Case

\title{
Pulmonary Resection for Lung Cancer Following Transcatheter Aortic Valve Implantation for Severe Aortic Valve Stenosis: A Case Report
}

\author{
Hiroaki Komatsu, MD, PhD, Nobuhiro Izumi, MD, PhD, Takuma Tsukioka, MD, PhD, \\ Kyukwang Chung, MD, PhD, Michihito Toda, MD, PhD, Kantaro Hara, MD, \\ and Noritoshi Nishiyama, MD, PhD
}

\begin{abstract}
An 82-year-old woman was referred to our hospital because of dyspnea on effort. Echocardiography revealed severe aortic valve stenosis (AS). Simultaneously, chest computed tomography (CT) revealed a 19-mm nodule in the lower lobe of the right lung, and bronchoscopic biopsy revealed adenocarcinoma. She underwent transcatheter aortic valve implantation (TAVI) for severe AS. Three weeks later, she underwent lower lobectomy of the right lung and mediastinal dissection for the lung cancer. Her postoperative course was good, and she was discharged 8 days postoperatively. In conclusion, we encountered a patient who successfully underwent pulmonary resection for lung cancer following TAVI for severe AS. We suggest that pulmonary resection following TAVI is an acceptable choice for lung cancer in patients with severe AS because of the lack of a need for cardiopulmonary bypass, the high safety and efficacy of two-stage therapy, and the short period between the two therapies.
\end{abstract}

Keywords: lung cancer, surgery, transcatheter aortic valve implantation, aortic valve stenosis

\section{Introduction}

The therapeutic strategy for lung cancer in patients with severe valvular heart disease is controversial. We herein report a patient who successfully underwent pulmonary resection for lung cancer following transcatheter aortic valve implantation (TAVI) for severe aortic valve stenosis (AS).

Department of Thoracic Surgery, Osaka City University Hospital, Osaka, Osaka, Japan

Received: February 4, 2018; Accepted: March 25, 2018

Corresponding author: Hiroaki Komatsu, MD, PhD. Department of Thoracic Surgery, Osaka City University Hospital, 1-4-3 Asahimachi, Abeno-ku, Osaka, Osaka 545-8585, Japan

Email: m1111276@med.osaka-cu.ac.jp

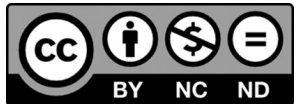

This work is licensed under a Creative Commons Attribution-NonCommercialNoDerivatives International License.

(C)2020 The Editorial Committee of Annals of Thoracic and Cardiovascular Surgery

\section{Case Report}

An 82-year-old woman was referred to our hospital because of dyspnea on effort. Echocardiography revealed severe AS with a tricuspid aortic valve. The mean and peak aortic valve pressure gradient were 55 and $98 \mathrm{mmHg}$, respectively. The aortic valve area was $0.6 \mathrm{~cm}^{2}$. Simultaneously, chest computed tomography (CT) revealed a 19 -mm nodule in the lower lobe of the right lung (Fig. 1), and lung cancer was suspected. Bronchoscopic biopsy revealed adenocarcinoma. Fluorodeoxyglucosepositron emission tomography (FDG-PET) and brain magnetic resonance imaging (MRI) revealed no distant metastases. The patient first underwent TAVI with the SAPIEN 3 transcatheter heart valve by a transfemoral approach for severe AS (Fig. 2). After TAVI, the mean and peak aortic valve pressure gradient decreased to 9 and $15 \mathrm{mmHg}$, respectively. There was no paravalvular 


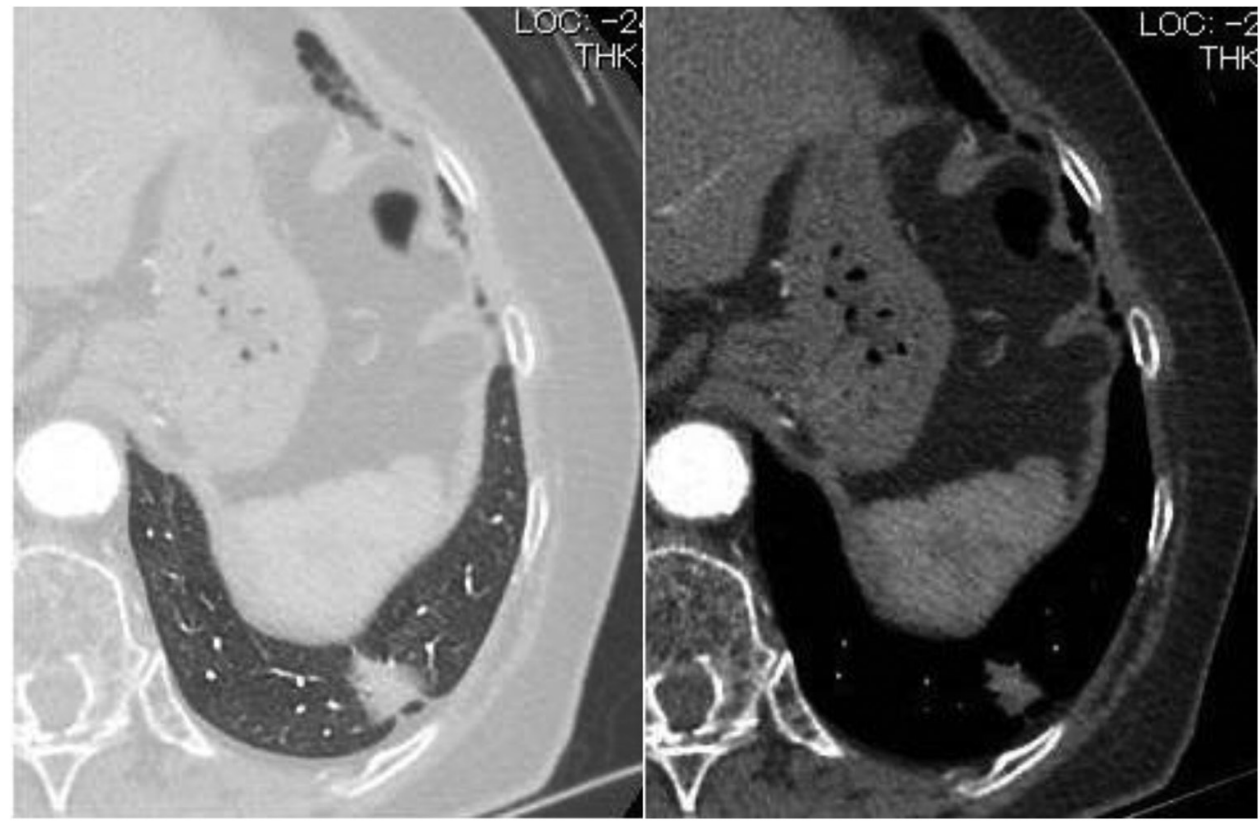

Fig. 1 Chest CT showing a 19-mm nodule in the lower lobe of the right lung. CT: computed tomography
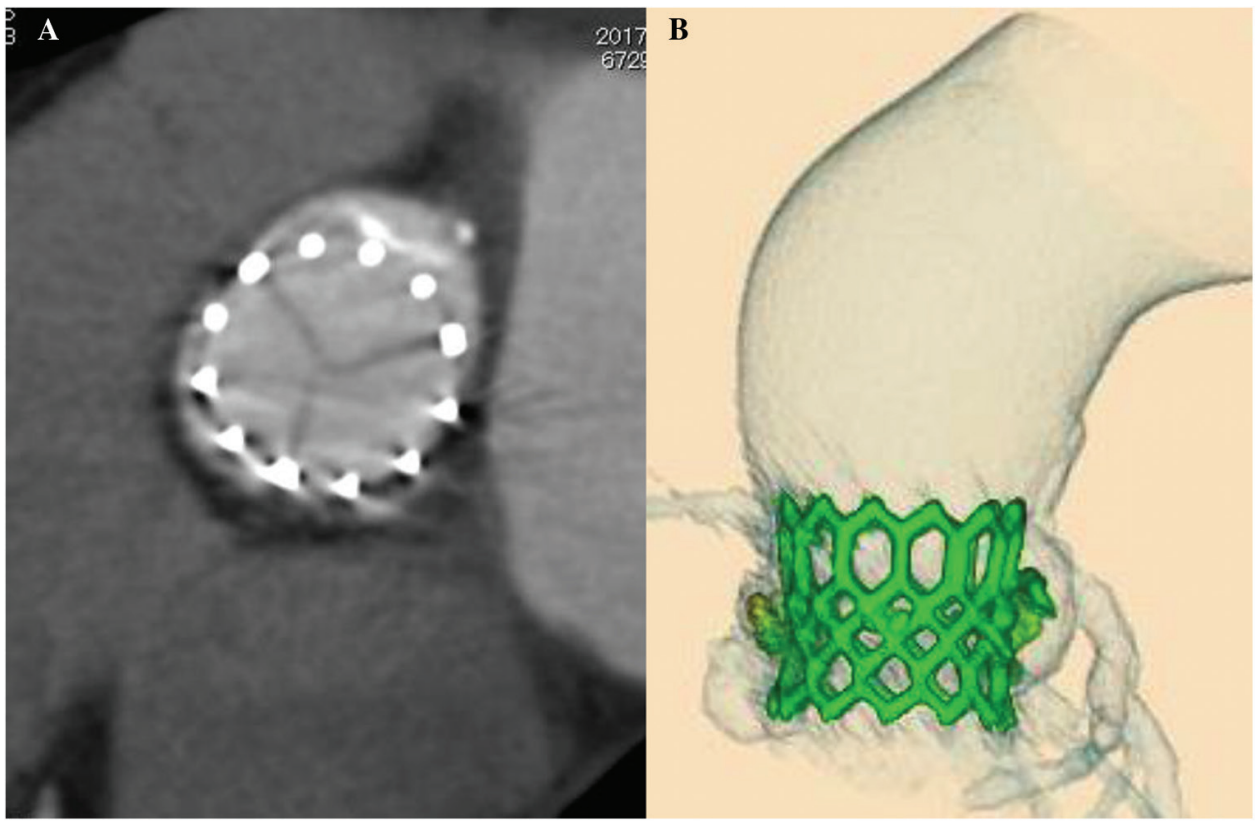

Fig. 2 Chest CT (A) and three-dimensional reconstruction (B) after TAVI showing successfully replaced transcatheter aortic valve. CT: computed tomography; TAVI: transcatheter aortic valve implantation

leakage. One day after TAVI, she began oral clopidogrel alone because of aspirin allergy. Her post-procedural course was uneventful. Three weeks later, she underwent lower lobectomy of the right lung and mediastinal dissection for the lung cancer. During the perioperative period of lung cancer, she discontinued the oral clopidogrel. Her postoperative course was again good. Histologic examination of the resected specimen revealed adenocarcinoma of the right lung and no metastases of the lymph nodes. The pathologic stage was T1bN0M0 stage IA2. She was discharged 8 days postoperatively. 


\section{Discussion}

TAVI has recently become an alternative to surgery for the treatment of AS in patients with high surgical risk. ${ }^{1)}$ Solitary pulmonary nodules are often detected incidentally on chest CT before therapy for severe valvular heart disease, such as TAVI for AS, and some of them are rarely diagnosed as lung cancer. ${ }^{2)}$ Conversely, preoperative examinations for lung cancer also occasionally reveal cardiovascular disease including valvular heart disease. In the recent report, of 1302 patients who underwent surgery for non-small-cell lung cancer, cardiovascular disease requiring invasive treatment was simultaneously discovered in $33(3 \%)$, including valvular heart disease in $6(0.5 \%))^{3)}$ Thus, in some patients, lung cancer and severe valvular heart disease occasionally coexist.

The therapeutic strategy for lung cancer in patients with severe valvular heart disease is controversial. According to the American College of Cardiology and American Heart Association (ACC/AHA) guidelines on perioperative cardiovascular evaluation and management of patients undergoing noncardiac surgery, valvular intervention before noncardiac surgery is effective in reducing perioperative risk for patients with standard indications for valvular intervention based on their symptoms and severity of stenosis or regurgitation. ${ }^{4)}$ In two-stage surgery (pulmonary resection following cardiac surgery), however, coexisting lung cancer might grow rapidly to the inoperative stage after the cardiac surgery. In addition, cardiac surgery with cardiopulmonary bypass may cause spread and dissemination of coexisting lung cancer.,5) The efficacy of TAVI is similar to that of surgery, ${ }^{1)}$ and it has fewer disadvantages with respect to the growth of coexisting lung cancer after therapy than cardiac surgery because TAVI requires a shorter recovery period until patients can undergo subsequent pulmonary resection than cardiac surgery. In fact, in the present patient, the period between TAVI and pulmonary resection was only 3 weeks, whereas the second surgery should be performed more than 6 weeks after the cardiac surgery with cardiopulmonary bypass whenever possible. ${ }^{7)}$ However, there are no data regarding the efficacy or safety of TAVI for patients with AS who are undergoing noncardiac surgery. Although one-stage surgery for cardiac surgery and pulmonary resection reportedly has acceptable operative morbidity and mortality, avoidance of cardiopulmonary bypass might help to decrease blood loss and ventilation requirements. ${ }^{8)}$

TAVI is associated with a possible higher risk of periprocedural stoke or transient ischemic attack than cardiac surgery at 30 days. ${ }^{9)}$ To limit the risk of cerebral embolization, ACC/AHA guidelines recommend clopidogrel $75 \mathrm{mg}$ daily for the first 6 months after TAVI in addition to life-long aspirin (75-100 mg daily). ${ }^{4)} \mathrm{How}-$ ever, no difference in net adverse clinical and cerebral events rate at 30 days was observed between aspirin only or dual-antiplatelet therapy following TAVI. ${ }^{10)}$ Thus, subsequent support for the guidelines on postTAVI antiplatelet therapy is currently insufficient. The present patient was treated by only clopidogrel as postTAVI antiplatelet therapy because of aspirin allergy. Although she discontinued it for the surgery of lung cancer even within 30 days after TAVI, she had no net adverse clinical and cerebral events.

We suggest that pulmonary resection following TAVI is an acceptable choice for lung cancer in patients with severe AS because of the lack of a need for cardiopulmonary bypass, high safety and efficacy of two-stage therapy, and the short period between the two therapies.

\section{Acknowledgment}

We thank Angela Morben, DVM, ELS, from Edanz Group for editing a draft of this manuscript.

\section{Disclosure Statement}

None declared.

\section{References}

1) Mack MJ, Leon MB, Smith CR, et al. 5-year outcomes of transcatheter aortic valve replacement or surgical aortic valve replacement for high surgical risk patients with aortic stenosis (PARTNER 1): a randomised controlled trial. Lancet 2015; 385: 2477-84.

2) Schmidt LH, Vietmeier B, Kaleschke G, et al. Thoracic malignancies and pulmonary nodules in patients under evaluation for transcatheter aortic valve implantation (TAVI): incidence, follow up and possible impact on treatment decision. PLoS ONE 2016; 11: e0155398.

3) Kanzaki R, Kimura T, Kawamura T, et al. Treatment of simultaneously discovered lung cancer and cardiovascular disease: a 20 -year single-institution experience. Surg Today 2017; 47: 726-32.

4) Fleisher LA, Fleischmann KE, Auerbach AD, et al. 2014 ACC/AHA guideline on perioperative cardiovascular evaluation and management of patients undergoing noncardiac surgery: a report of the American college of cardiology/American heart association task force on practice guidelines. J Am Coll Cardiol 2014; 64: e77-137. 
5) Pinto CA, Marcella S, August DA, et al. Cardiopulmonary bypass has a modest association with cancer progression: a retrospective cohort study. BMC Cancer 2013; 13: 519.

6) Hasegawa S, Otake Y, Bando T, et al. Pulmonary dissemination of tumor cells after extended resection of thyroid carcinoma with cardiopulmonary bypass. J Thorac Cardiovasc Surg 2002; 124: 635-6.

7) Crawford ES, Morris GC, Howell JF, et al. Operative risk in patients with previous coronary artery bypass. Ann Thorac Surg 1978; 26: 215-21.
8) Danton MH, Anikin VA, McManus KG, et al. Simultaneous cardiac surgery with pulmonary resection: presentation of series and review of literature. Eur J Cardiothorac Surg 1998; 13: 667-72.

9) Miller DC, Blackstone EH, Mack MJ, et al. Transcatheter (TAVR) versus surgical (AVR) aortic valve replacement: occurrence, hazard, risk factors, and consequences of neurologic events in the PARTNER trial. J Thorac Cardiovasc Surg 2012; 143: 832-43.e13.

10) Hassell ME, Hildick-Smith D, Durand E, et al. Antiplatelet therapy following transcatheter aortic valve implantation. Heart 2015; 101: 1118-25. 